\title{
mRNA expression of interleukins and Th1/Th2 imbalance in patients with pulmonary embolism
}

\author{
QIANGLIN DUAN*, WEI LV*, LEMIN WANG, ZHU GONG, QIANG WANG, HAOMING SONG and HAO WANG \\ Department of Cardiology, Tongji Hospital of Tongji University, Shanghai 200065, P.R. China
}

Received May 24, 2012; Accepted October 18, 2012

DOI: $10.3892 / \mathrm{mmr} .2012 .1142$

\begin{abstract}
Few studies have investigated the changes of Th1- and Th2-type cytokines in pulmonary embolism (PE) patients. In this study, the gene expression of interleukins and the balance of Th1- and Th2-type cytokines in the peripheral blood mononuclear cells (PBMCs) of PE patients and controls were investigated. A total of $20 \mathrm{PE}$ patients and 20 genderand age-matched controls were included in the study. Human cDNA microarray analysis was used to detect the differences in cytokine gene expression between the two groups and a random variance model corrected t-test was used to analyze the statistical data. In comparison with the controls, 12 genes were found to be downregulated, specifically IL1A, IL9, IL17B, IL19, IL23A, IL25 (P<0.05), IL2, IL3, IL13, IL22, IL24 and IL31 $(\mathrm{P}<0.01)$, and 2 genes were found to be upregulated, specifically IL10 and IL28A, in the PE patients. The expression levels of IFN- $\gamma$ and IL2 mRNA in the PE patients were significantly lower than those in the control group $(\mathrm{P}<0.01)$, while the IL20 mRNA expression levels were significantly upregulated $(\mathrm{P}<0.01)$. We conclude that there are significant differences in interleukin gene expression between the PE patients and the control group. A shift of the Th1/Th2 balance comprising enhanced Th2 activity and reduced Th1 activity in the PE patients is also demonstrated.
\end{abstract}

\section{Introduction}

Venous thromboembolism (VTE) includes PE thrombosis (DVT) and pulmonary embolism (PE) (1) and PE comprises acute pulmonary embolism (APE) and chronic thromboembolic pulmonary hypertension (CTEPH). VTE may be acquired or inherited, but is most commonly acquired, and its prophylaxis is not satisfactory. PE, with its high incidence,

Correspondence to: Professor Lemin Wang, Department of Cardiology, Tongji Hospital of Tongji University, 389 Xincun Road, Putuo, Shanghai 200065, P.R. China

E-mail:wanglemin@tongji.edu.cn

${ }^{*}$ Contributed equally

Key words: pulmonary embolism, interleukins, genechip, Th1/Th2 high mortality and high rates of missed diagnosis and misdiagnosis, has become a major health problem worldwide (2). The immune system comprises innate and adaptive defenses and the latter involves cell-mediated and humoral immune components. We have previously reported that patients with CTEPH had reduced T cell-mediated immune function (3) and we have also demonstrated that VTE is a systemic disease, the incidence of which is related to viral infection (4). Interleukins play an important role in immune regulation. Th1 cells, which produce IL2 and IFN- $\gamma$, chiefly mediate cellular immune responses, while Th2 cells, which secrete IL4, IL6 and IL10, chiefly mediate humoral immune responses (5). In the current study, human cDNA microarray analysis was used to detect the expression of interleukin and IFN- $\gamma$ genes in PBMCs and changes in the acquired immune functions of PE patients and the control group.

\section{Patients and methods}

Patients. A total of 20 patients with PE were recruited from the Tongji Hospital of Tongji University from 2007 to 2008. A diagnosis of PE required any two of the following three criteria: i) selectivity pulmonary angiography shows pulmonary artery obstruction or filling defect; ii) lung ventilation/perfusion scan shows single or multiple blood perfusion defect, normal or abnormal ventilation and V/Q does not match; iii) clinical diagnosis: risk factors for PE are present and other cardiovascular diseases may be excluded by clinical performance, electrocardiogram and chest film; arterial blood gas analysis suggests hypoxemia and hypocapnia; and D-dimer detection, echocardiography and/or chest computed tomography support PE diagnosis. We selected 20 patients admitted to our Department of Cardiology over the same time period to act as controls. The patients were divided into two groups: i) a PE patient group comprising 20 patients (11 males and 9 females) with a mean age of $70 \pm 14$ (44-89) years, including 3 cases of CTEPH; and ii) a control group comprising 20 patients (11 males and 9 females) without PE, DVT, arterial thrombosis or congenital coagulation abnormality, with a mean age of $72 \pm 14$ (44-91) years, which were matched by gender and age with the PE group. No statistically significant differences were observed between the ages of the two groups $(\mathrm{P}>0.05)$. The clinical trial was approved by the Ethics Committee of Tongji University and all participants provided their written informed consent. 
Gene expression profiling. Agilent G4112A Whole Human Genome Oligo microarrays were purchased from Agilent Technologies (Palo Alto, CA, USA). The microarray comprises 44,290 spots, including 41,675 genes or transcripts, 314 negative control spots, 1,924 positive control spots and 359 blank spots. The functions of $>70 \%$ of genes in the microarray are known. All patients were subjected to microarray analysis.

Total RNA isolation. Peripheral blood samples $(5 \mathrm{ml})$ anticoagulated with EDTA were drawn from the patients suspected of having PE immediately after admittance to the hospital and from the patients without PE. Mononuclear cells were obtained through density gradient centrifugation with Ficoll solution and the remaining red blood cells were destroyed with erythrocyte lysis buffer (Qiagen, Hilden, Germany). Total mononuclear cell RNA was extracted with TRIzol (Invitrogen, Carlsbad, CA, USA) and purified using a Qiagen RNeasy column (Qiagen) according to the manufacturer's instructions. The isolated total RNA was identified and quantified using a Nanodrop ND-1000 spectrophotometer (Nanodrop Technology, Cambridge, UK).

Detection of gene expression. Total RNA $(\sim 1 \mu \mathrm{g})$ was reversetranscribed into double-stranded cDNA. After purification, in vitro amplification was performed using the Agilent low RNA input linear amplification kit (Agilent Technologies) and modified UTP [aaUTP, 5-(3-aminoally1)-UTP] was used to replace UTP. The integrated aaUTP may interact with $\mathrm{Cy} 3$ NHS ester to form fluorescent products which are then used for hybridization. The integration rate of fluorescence was determined using the Nanodrop ND-1000 spectrophotometer. A hybridization mixture was prepared using an Agilent oligonucleotide microarray In situ Hybridization Plus kit. The fluorescent products $(\sim 750 \mathrm{ng})$ were fragmented at $60^{\circ} \mathrm{C}$ and hybridization was conducted on Human Whole-Genome 60 -mer oligo chips (G4112F, Agilent Technologies) at $60^{\circ} \mathrm{C}$ for $17 \mathrm{~h}$ at $10 \mathrm{rpm}$. After hybridization, the chips were washed with Agilent Gene Expression wash buffer according to the manufacturer's instructions. Original signals were obtained with Agilent scanner and Feature Extraction software. The standardization of the original signals was carried out by the RMA standardized method and standardized signal values were used for the screening of differentially expressed genes.

$R T$-PCR. Three genes, CCL3L3, ZNF683 and LBA1, were selected from the microarray and their expression was confirmed by RT-PCR. From the differentially expressed genes, 3 genes were randomly selected and subjected to RT-PCR together with the housekeeping gene (GAPDH). The relative expression levels were expressed as the expression levels of the target genes normalized to the expression level of GAPDH $\left(2^{-\Delta \Delta \mathrm{Ct}}\right)$. Melting curve and $2^{-\Delta \Delta \mathrm{Ct}}$ methods were used to compare the differences in the expression levels between the control and PE groups. The results from the RT-PCR procedure were consistent with those from the microarray analysis.

Statistical analysis. Agilent Feature Extraction software was used to collect original data from the microarray which was then analyzed using the robust multichip average (RMA) algorithm. Gene intensity data for the PE and control groups were
Table I. Interleukin gene expression in the pulmonary embolism (PE) and control groups.

\begin{tabular}{|c|c|c|}
\hline Gene & PE group & Control group \\
\hline IL1A & $4.47 \pm 0.52^{\mathrm{a}}$ & $4.98 \pm 0.75$ \\
\hline IL1B & $14.79 \pm 0.86$ & $14.56 \pm 0.69$ \\
\hline IL2 & $4.47 \pm 0.38^{b}$ & $4.99 \pm 0.71$ \\
\hline IL3 & $4.37 \pm 0.53^{b}$ & $5.10 \pm 0.99$ \\
\hline IL4 & $6.39 \pm 1.32$ & $6.92 \pm 1.51$ \\
\hline IL5 & $5.10 \pm 0.96$ & $4.86 \pm 0.71$ \\
\hline IL6 & $6.66 \pm 0.71$ & $6.55 \pm 0.47$ \\
\hline IL7 & $8.40 \pm 0.93$ & $8.62 \pm 0.60$ \\
\hline IL8 & $11.27 \pm 1.86$ & $12.17 \pm 1.62$ \\
\hline IL9 & $4.51 \pm 0.53^{\mathrm{a}}$ & $5.20 \pm 1.11$ \\
\hline IL10 & $7.68 \pm 0.72^{b}$ & $6.69 \pm 0.48$ \\
\hline IL11 & $5.11 \pm 0.80$ & $5.29 \pm 0.80$ \\
\hline IL12A & $7.03 \pm 0.74$ & $7.36 \pm 0.53$ \\
\hline IL12B & $5.46 \pm 1.48$ & $5.39 \pm 0.94$ \\
\hline IL13 & $4.58 \pm 0.55^{\mathrm{b}}$ & $5.44 \pm 1.09$ \\
\hline IL15 & $12.21 \pm 0.56$ & $12.37 \pm 0.43$ \\
\hline IL16 & $10.71 \pm 0.47$ & $10.62 \pm 0.31$ \\
\hline IL17A & $7.02 \pm 0.37$ & $7.01 \pm 0.81$ \\
\hline IL17B & $4.33 \pm 0.37^{\mathrm{a}}$ & $4.99 \pm 1.27$ \\
\hline IL17C & $6.07 \pm 0.70$ & $6.03 \pm 0.68$ \\
\hline IL17D & $4.90 \pm 0.98$ & $5.19 \pm 0.78$ \\
\hline IL17F & $5.78 \pm 1.02$ & $5.87 \pm 1.18$ \\
\hline IL18 & $11.02 \pm 0.53$ & $10.79 \pm 0.46$ \\
\hline IL19 & $4.90 \pm 0.46^{\mathrm{a}}$ & $5.42 \pm 0.91$ \\
\hline IL20 & $4.78 \pm 0.85$ & $5.22 \pm 0.76$ \\
\hline IL21 & $5.56 \pm 0.47$ & $5.64 \pm 0.78$ \\
\hline IL22 & $4.18 \pm 0.42^{b}$ & $4.78 \pm 0.79$ \\
\hline IL23A & $10.68 \pm 0.88^{a}$ & $11.19 \pm 0.59$ \\
\hline IL24 & $5.15 \pm 0.35^{\mathrm{b}}$ & $5.53 \pm 0.37$ \\
\hline IL25 & $4.62 \pm 0.50^{\mathrm{a}}$ & $5.26 \pm 0.93$ \\
\hline IL26 & $5.37 \pm 0.63$ & $5.78 \pm 1.05$ \\
\hline IL27 & $12.26 \pm 1.04$ & $12.13 \pm 1.03$ \\
\hline IL28A & $9.38 \pm 0.63^{\mathrm{a}}$ & $8.84 \pm 0.71$ \\
\hline IL28B & $5.00 \pm 1.23$ & $5.65 \pm 1.76$ \\
\hline IL29 & $7.05 \pm 0.74$ & $7.45 \pm 0.57$ \\
\hline IL31 & $4.18 \pm 0.44^{\mathrm{b}}$ & $4.77 \pm 0.72$ \\
\hline IL32 & $15.06 \pm 0.65$ & $15.20 \pm 0.61$ \\
\hline
\end{tabular}

PE, pulmonary embolism. ${ }^{\mathrm{a}} \mathrm{P}<0.05,{ }^{\mathrm{b}} \mathrm{P}<0.01$.

compared using the random variance model corrected t-test. Differentially expressed genes were identified from whole genomes. $\mathrm{P}<0.05$ was considered to indicate a statistically significant result.

\section{Results}

Differential expression of interleukins. A total of 37 interleukin genes were detected (Table I). In comparison with the control, the expression levels of 12 genes were downregulated 

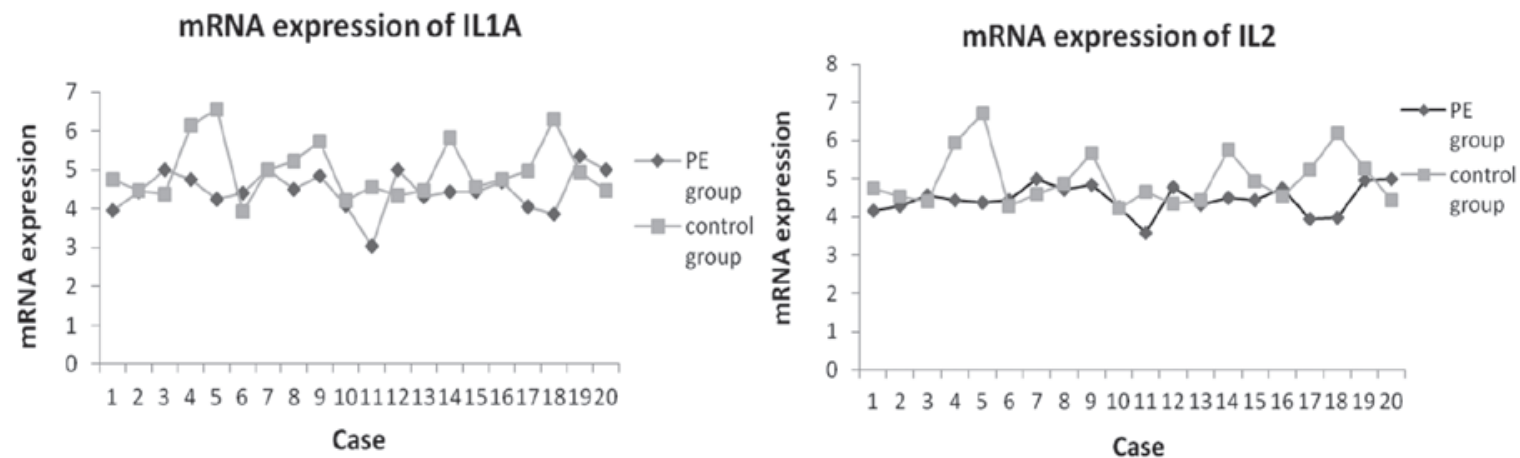

mRNA expression of IL3
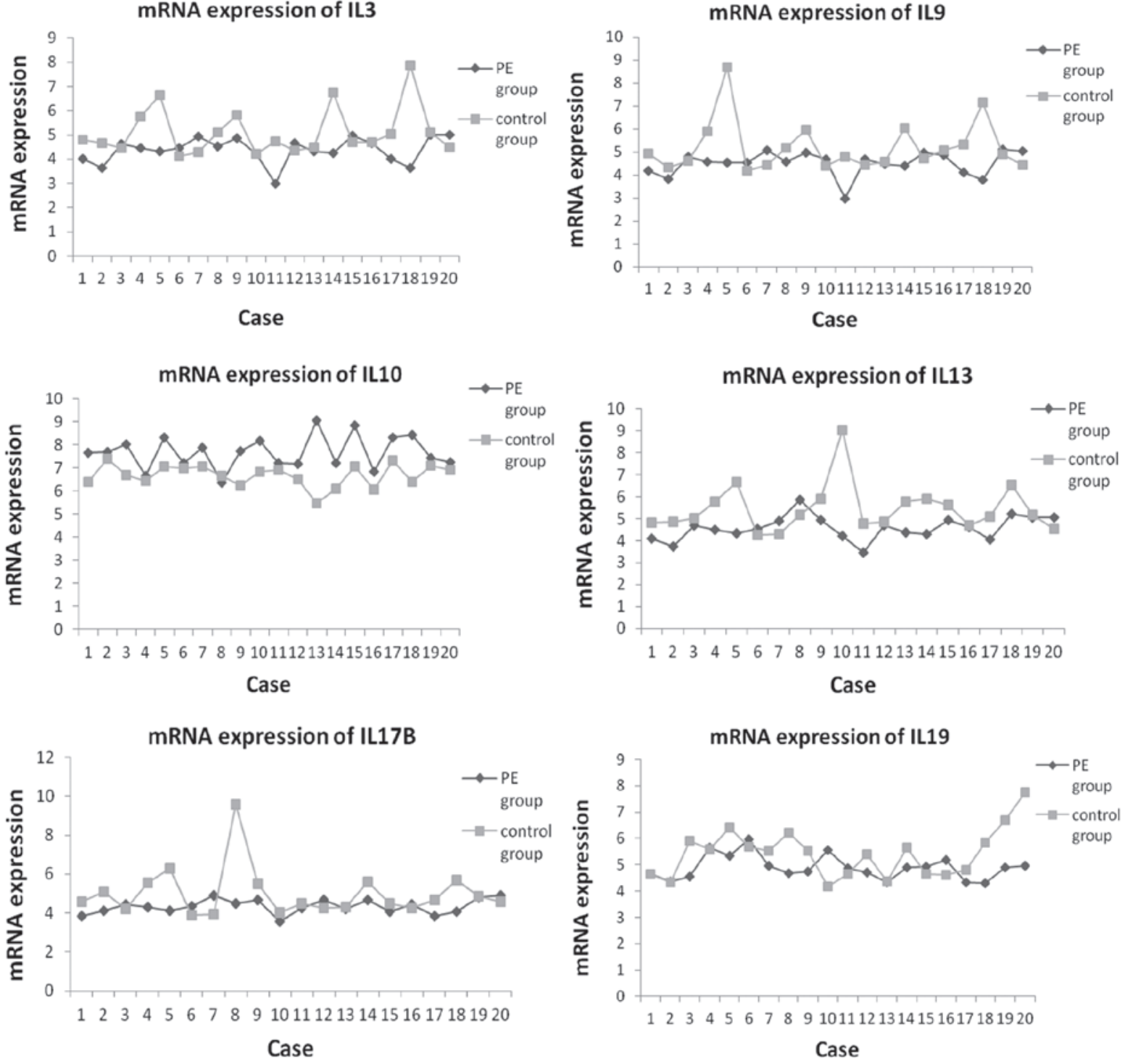

Figure 1. Expression of IL1A, IL2, IL3, IL9, IL10, IL13, IL17B and IL19 mRNA in pulmonary embolism (PE) patients and controls.

(Figs. 1-3), specifically IL1A, IL9, IL17B, IL19, IL23A, IL25 $(\mathrm{P}<0.05)$, IL2, IL3, IL13, IL22, IL24 and IL31 ( $\mathrm{P}<0.01)$, and those of 2 of the genes were upregulated, specifically IL10 and IL28A $(\mathrm{P}<0.05)$, in the PE patients.

mRNA expression of Th1 and Th2 cytokines. The mRNA expression levels of the Th1-type cytokines IFN- $\gamma$ and IL2 in the PE patients were significantly lower than those in the control group $(\mathrm{P}<0.01)$, while the mRNA expression levels of the Th2-type cytokine IL10 were significantly increased $(\mathrm{P}<0.01$; Tables II and III).

\section{Discussion}

Human cDNA microarray analysis was used to detect the expression of interleukin and IFN- $\gamma$ genes in PBMCs from the 

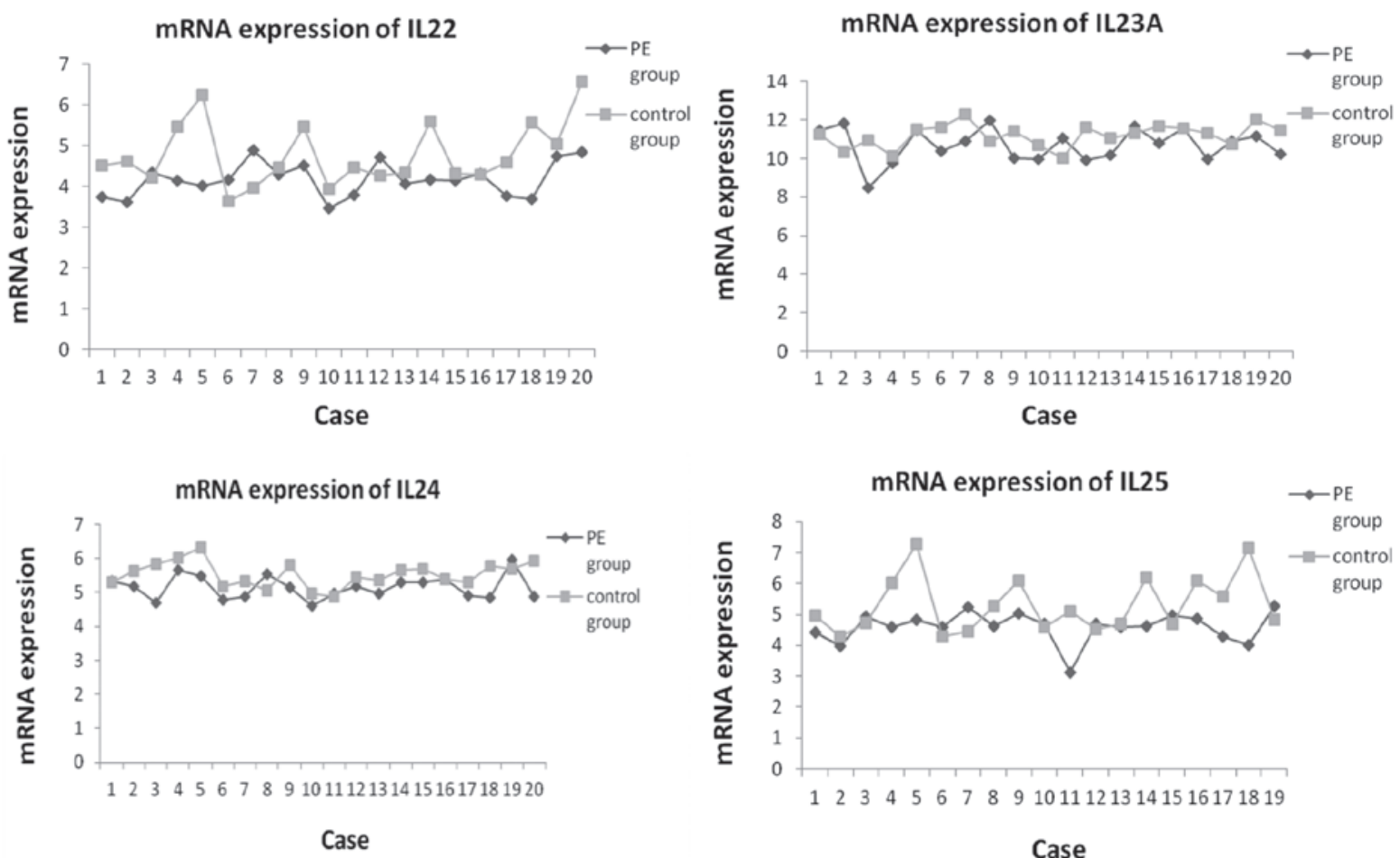

mRNA expression of IL28A
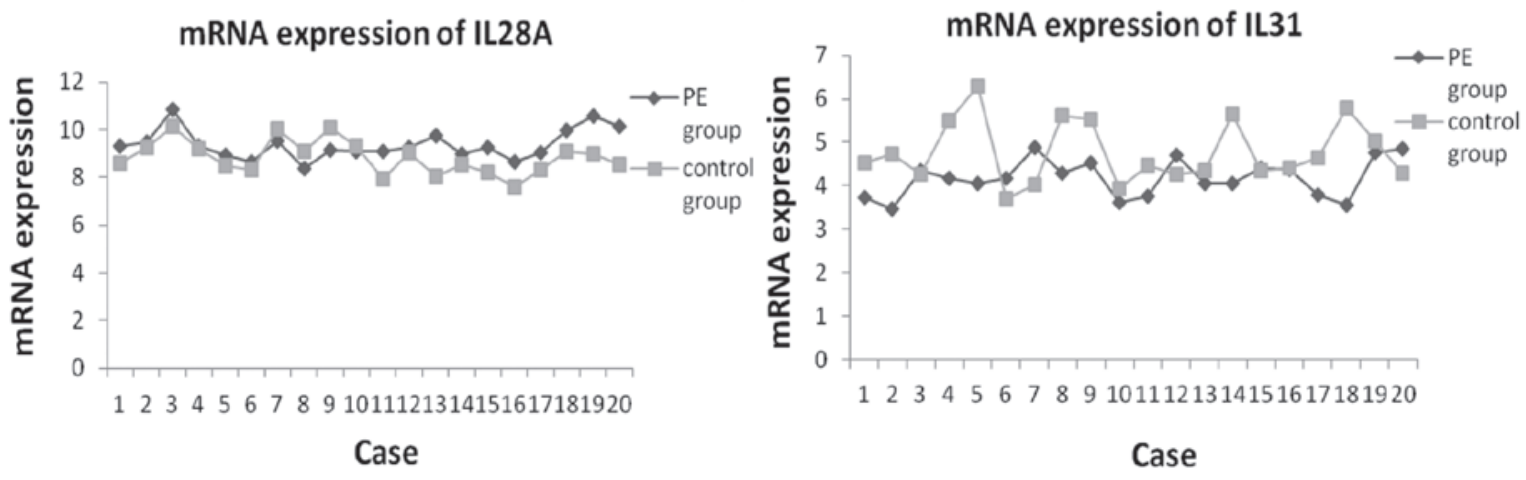

Figure 2. Expression of IL22, IL23A, IL243, IL25, IL28A and IL31 mRNA in the pulmonary embolism (PE) patients and controls.

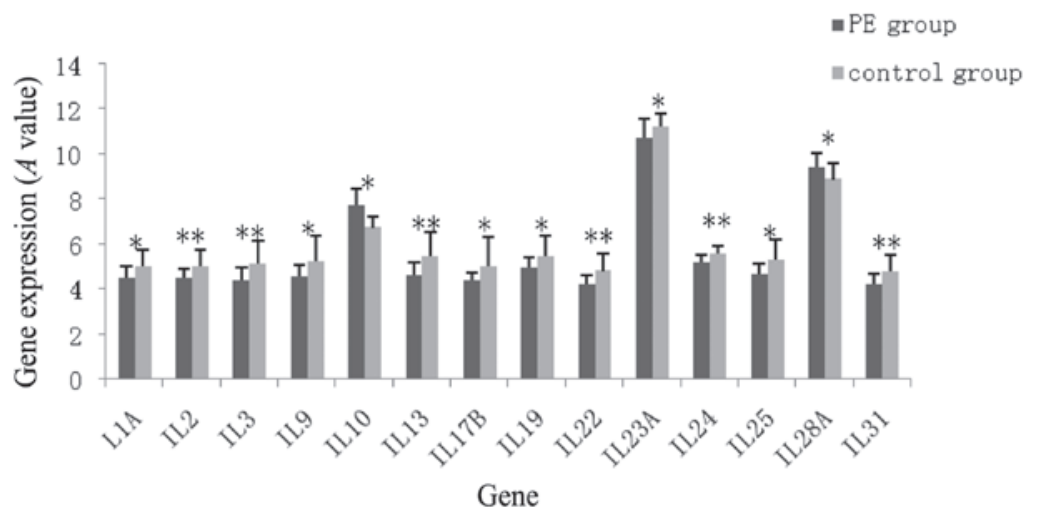

Figure 3. Differentially expressed interleukin genes between the pulmonary embolism (PE) and control groups. ${ }^{*} \mathrm{P}<0.05,{ }^{* *} \mathrm{P}<0.01$.

PE and control groups. The differential expression of interleukins and the Th1/Th2 balance were analyzed. The cytokines included IL2 and IFN- $\gamma$ as Th1-type cytokines and IL4, IL6, IL10 as Th2-type cytokines.
Th precursor cells may be differentiated into Th1 or Th2 cells. The direction of differentiation is significantly influenced by a variety of factors, including the cytokine environment, altered peptide ligands as antigens, varying 
Table II. Expression of Th1-type cytokine genes in the pulmonary embolism (PE) and control groups.

\begin{tabular}{lcc}
\hline Group & IFN- $\gamma$ & IL2 \\
\hline PE group & $9.45 \pm 0.94^{\mathrm{a}}$ & $4.47 \pm 0.38^{\mathrm{a}}$ \\
Control group & $10.21 \pm 0.76$ & $4.99 \pm 0.71$ \\
\hline
\end{tabular}

${ }^{\mathrm{a}} \mathrm{P}<0.01$.

Table III. Expression of Th2-type cytokine genes in the pulmonary embolism (PE) and control groups.

\begin{tabular}{lccc}
\hline Group & IL4 & IL6 & IL10 \\
\hline PE group & $6.39 \pm 1.32$ & $6.66 \pm 0.71$ & $7.68 \pm 0.72^{\text {a }}$ \\
Control group & $6.92 \pm 1.51$ & $6.55 \pm 0.47$ & $6.69 \pm 0.48$ \\
\hline
\end{tabular}

${ }^{\mathrm{a}} \mathrm{P}<0.01$.

quantities of antigen and altered co-stimulatory signals (6). Th1 lymphocytes secrete IL2, IFN- $\gamma$ and IL12, which regulate cell-mediated immunity by promoting the differentiation, maturation and proliferation of $\mathrm{T}$ lymphocytes and increasing the activity of NK cells. Th2 lymphocytes secrete IL4, IL6 and IL10 and promote the differentiation, maturation and proliferation of B lymphocytes and the generation of antibodies. Changes in the levels of Th1 and Th2 cytokines reflect the immune balance. A shift of the Th1/Th2 balance may cause an immunological regulation function disorder.

The immune system needs to maintain a balance since imbalance will cause it to over- or under-respond. In the adaptive immune system, cell-mediated immunity and humoral immunity also require balance and this is primarily regulated by Th1 and Th2 cytokines.

In the current study, the mRNA expression levels of the Th1 cytokines IFN- $\gamma$ and IL2 in the PE patients were significantly downregulated $(\mathrm{P}<0.01)$, while the mRNA expression levels of the Th2 cytokine IL10 were significantly upregulated, suggesting reduced Th1 activity and enhanced Th2 activity. The shift of the Th1/Th2 balance indicates a disorder of the immunological regulation function. Downing et al have reported elevated IL10 in the vein wall during venous thrombosis (7) and elevated levels of IL6 have been reported in DVT (8). These studies show increased levels of Th2 cytokines and enhancement of the humoral immune response in VTE, which supports our results.

We have previously reported that the numbers of $\mathrm{CD}^{+}$ and $\mathrm{CD}^{+} \mathrm{T}$ cells in patients with CTEPH are significantly reduced (3) and that virus-like structures were synthesized and released in a patient with pulmonary hypertension (9). We have also previously found that $\mathrm{T}$ cell and NK cell immunity function-associated genes were downregulated in patients with $\mathrm{PE}(10)$, and that $\mathrm{CD}^{+} \mathrm{CD}^{+} \mathrm{T}$ cell immunity is dysfunctional in acute PE patients (11). These studies demonstrate a compromised cell-mediated immunity. In the current study, a shift of the Th1/Th2 balance along with enhanced Th2 activity in PE patients was demonstrated, indicating that cell-mediated immunity was compromised. The result is consistent with the reduced $\mathrm{T}$ cell function reported in the aforementioned studies

Our findings demonstrate the association between symptomatic VTE and adaptive immunity. The imbalance of Th1/Th2 manifests as reduced cell-mediated immunity and increased humoral immunity, which indicates that the immune system plays a role in the regulation of the immune balance. The upregulation of humoral immunity may be the result of the regulation of the immune system to establish a new balance. The immune system comprises two major parts, the innate immune system and the adaptive immune system, which defend against invading organisms. The function of $\mathrm{T}$ cells and NK cells in cell-mediated immunity is mainly antiviral, while the principal function of B cells in humoral immunity is the generation of antibodies. When there is a disturbance in immune function, the ratio of Th1/Th2 shifts and the body is susceptible to infection. Infection is a risk factor for VTE (12), therefore individuals who are vulnerable to infection may be susceptible to VTE.

\section{Acknowledgements}

This study was supported by the National Natural Science Foundation of China (30871080).

\section{References}

1. Imberti D, Ageno W, Manfredini R, et al: Interventional treatment of venous thromboembolism: a review. Thromb Res 129: 418-425, 2012.

2. Heil JA: The epidemiology of venous thromboembolism in the community. Arterioscler Thromb Vasc Biol 28: 370-372, 2008.

3. Haoming S, Lemin $\mathrm{W}$, Zhu G, et al: T cell-mediated immune deficiency or compromise in patients with CTEPH. Am J Respir Crit Care Med 183: 417-418, 2011.

4. Xiang-Hua Y, Le-Min W, Ai-Bin L, et al: Severe acute respiratory syndrome and venous thromboembolism in multiple organs. Am J Respir Crit Care Med 182: 436-437, 2010.

5. Xiao W, Yu H and Zheng C: The imbalance of Th1/Th2 cytokine expression in peripheral blood mononuclear cell from asthmatic patients and the effect of erythromycin on these cytokines. Zhonghua Jie He He Hu Xi Za Zhi 23: 347-350, 2000 (In Chinese).

6. Constant SL and Bottomly K: Induction of Th1 and Th2 $\mathrm{CD}^{+}{ }^{+} \mathrm{T}$ cell responses: the alternative approaches. Annu Rev Immunol 15: 297-322, 1997.

7. Downing LJ, Strieter RM, Kadell AM, et al: IL-10 regulates thrombus-induced vein wall inflammation and thrombosis. J Immunol 161: 1471-1476, 1998.

8. Fox EA and Kahn SR: The relationship between inflammation and venous thrombosis. A systematic review of clinical studies. Thromb Haemost 94: 362-365, 2005.

9. Wang L, Gong Z, Liang A, et al: Compromised T-cell immunity and virus-like structure in a patient with pulmonary hypertension. Am J Respir Crit Care Med 182: 434-435, 2010.

10. Gong Z, Liang AB, Wang LM, et al: The expression and significance of immunity associated genes mRNA in patients with pulmonary embolism. Zhonghua Nei Ke Za Zhi 48: 666-669, 2009 (In Chinese).

11. Wang L, Song H, Gong Z, Duan Q and Liang A: Acute pulmonary embolism and dysfunction of $\mathrm{CD}^{+} \mathrm{CD}^{+} \mathrm{T}$ cell immunity. Am J Respir Crit Care Med 184: 1315, 2011.

12. Schmidt M, Horvath-Puho E, Thomsen RW, Smeeth L and Sørensen HT: Acute infections and venous thromboembolism. J Intern Med 271: 608-618, 2012. 